is distinctly higher than the accepted $\mathrm{C}=12 \cdot 00$. Nevertheless, this high result, which indicates about one per cent of the 13 isotope, is in agreement with the spectroscopic work of Jenkins and Ornstein ${ }^{2}$, and the value of $\mathrm{C}=12.011$ of Woodhead and Whytlaw-Gray ${ }^{3}$ Indeed, the agreement between our values from two entirely different gases and those of the other workers is so close that it seems very improbable that they are in error, and consequently that the true atomic weight of carbon is $12 \cdot 01$.

The value of $F=18.995$ does not agree with the preliminary value of $\mathrm{F}=19 \cdot 01$ which we published in these columns ${ }^{4}$, using methyl fluoride. Consequently, these measurements have recently been repeated with the new apparatus. The new data lead to an atomic weight of $\mathrm{F}=18.995$ and a compressibility of $A_{21{ }^{\circ} \mathrm{C}}=0 \cdot 0090$. As would be expected, the adsorption error does not affect the compressibility to any marked extent. This last figure is in agreement with $A_{21}{ }^{\circ} \mathrm{C} .=0.0088$, measured on the Andrew's apparatus. It leads to $A_{0}{ }^{\circ} \mathrm{C} .=0 \cdot 0117$, which does not confirm the value of Moles and Batuecas ${ }^{5}$. Consequently, our previous criticisms ${ }^{6}$ still hold good.

Chemistry Department,

University of Leeds. Jan. 4.

${ }^{1}$ Cawood and Patterson, J. Chem. Soc., 156, 619; 1933.

2 Proc. K. Acad. Wetench. Amsterdam, 33, 1212; 1932.

3. Chem. Soc., 209, 846; 1933.

'J. Chem. Soc., 209, 846; 1933.
s $J$. Chim. Phys., 18, 353; NATure, 128, 375; 1920.

'Patterson and Cawood, Nature, 129, 245 ; 1932.

\section{Effect of Temperature on the Absorption of Crystals} in the Infra-Red

IN a recent publication, Matossi and his collaborators $^{1}$ have investigated experimentally the influence of temperature on the absorption of crystals in the infra-red; this is to some degree an extension of the classical work of Rubens and Hertz ${ }^{2}$.

A rather interesting point raised is that the funda. mental faature of the earlier work-the distinction between regions sensitive and insensitive to tem. perature-may be spurious, and due to the shift with temperature of the band as a whole.

It is possible to say, however, that the distinction drawn by Rubens and Hertz does exist, though not in the form suggested by these investigators-that the 'inner' vibrations are the insensitive, and 'outer' vibrations the sensitive ones. The theory of the damping of the infra-red vibrations ${ }^{3}$ shows that this is due to the coupling between the main vibrations and combinations of two other normal vibrations; and, further, that $(a)$ the absorption on the short wave-length of the main vibration $\left(\lambda_{0}\right)$ up to a point roughly $\lambda_{0} / \sqrt{ } 2$, is due to summation tones, and (b) the absorption on the long wave-length side is due to difference tones.

In the language of the quantum theory, the summation tones are produced by jumps from lower to higher quantum levels, whereas in a difference tone one of the jumps is from an excited to a lower level. It is obvious that, at very low temperatures, very few oscillators are in an excited state, hence the absorption due to difference tones must decrease to zero as the temperature decreases to zero.

It is also clear that absorption due to summation tones must be relatively insensitive to temperature, since at very low temperatures, where most oscillators are in the ground state, we can still have jumps from the ground state to excited states.

Hence the absorption on the short wave-length side of the main vibration should be relatively insensitive, that on the long wave-length side sensitive, to temperature. The distinction is obviously of the same kind as between the Stokes and anti-Stokes lines in the Raman effect*.

There is unfortunately no experimental data in the region between $\lambda_{0}$ and $\lambda_{0} \sqrt{ } 2$, where one would expect the really interesting effects; it is to be hoped that experimenters will investigate this region as well as the other regions.

A more detailed treatment of the absorption in the infra-red will be given in a forthcoming paper. M. BLACKMAN.

Mathematics Department,

Royal College of Science,

South Kensington, S.W.7. Dec. 19.

${ }^{2}$ F. Matossi and H. Brix, $Z$. Phys., 92, 303; 1934. F. Matossi and H. Kindler, $Z$. Phys., 92,$312 ; 1934$

2 H. Rubens and G. Hertz, Berlin Ber., $256 ; 1912$

'M. Born and M. Blackman, Z. Phy8., 82, 551; 1933. M. Blackman, Z. Phys., 88, 421 ; 1933. See also W. Pauli, Verh. d. D. Phys., o, 10 ; 1925

A. Smekal, Naturw., 11, 873; 1923.

Surface Tension of Urine during the Menstrual Cycle

We have recently earried out in this laboratory surface tension measurements in connexion with the excretion of capillary-active substances in the urine of the normal human female. Over a period of twenty-eight days the surface tension of the first morning specimen each day was determined, and the centrifuged deposit examined microscopically for blood corpuscles, so that the onset of menstruation could be ascertained as early as possible. A typical curve of the variation is shown in Fig. 1, and from this the following interesting observations can be made.

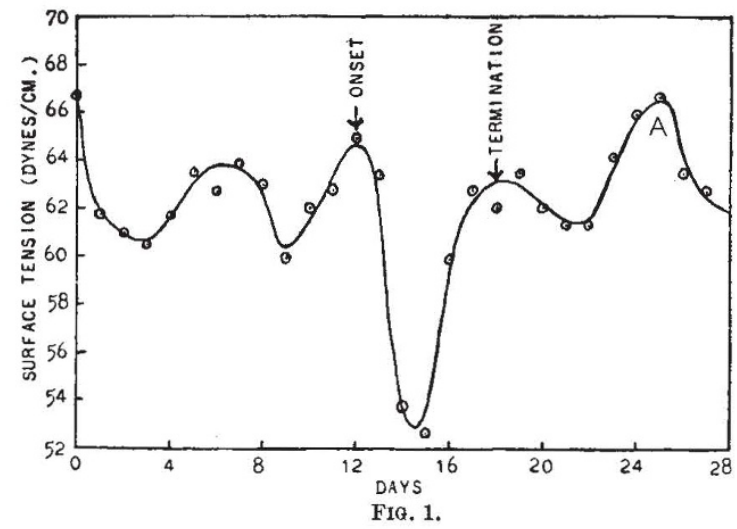

The curve consists of four phases corresponding in temporal relation to the four divisions into whichon physiological evidence-the human period can be divided, namely, resting, constructive, destructive and repair stages.

As regards the length and rhythm of cycle, it ap. pears that the measurement of surface tension possibly provides a new means of estimating these hitherto inaccessible factors. For example, the curve shown is one of a cycle of length 25 days and of normal rhythm.

At the loop $A$ on the diagram where one cycle ends 